\title{
First baby born out of a transplanted uterus
}

\author{
Jan Deprest • Jacques Donnez
}

Published online: 1 November 2014

(C) Springer-Verlag Berlin Heidelberg 2014

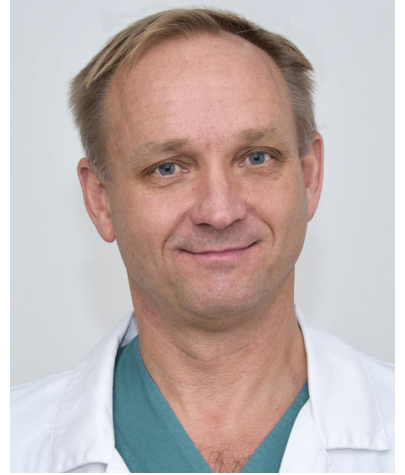

Mats Brännström

\section{Follow up on a ESGE annual congress key-note lecture by Mats Brönström}

Only weeks after Mats Brännström gave his key-note lecture at the annual ESGE conference in Brussels, the Lancet publishes a case observation of the first live born baby following uterine transplantation [1]. On his last slide, he forecasted live births within a year. Most must have thought this was a realistic forecast; the audience could not know that his confidence may have indicated that an imminent report was still under embargo, hence too early to share this news with the nearly 2000 congress participants. The first live born baby after uterine transplantation rewards over 15 years of meticulous and robust research by the team from Göteborg, Sweden.

\footnotetext{
J. Deprest $(\bowtie)$

Department of Obstetrics and Gynecology, University Hospitals Leuven, KU Leuven, Leuven, Belgium

e-mail: jan.deprest@uzleuven.be

J. Donnez

Obstetrics and Gynaecology, Catholic University of Louvain, Society for Research into Infertility, Brussels, Belgium
}

The enterprise was considered as the last frontier in fertility treatment, hence breached in 2014. The many women facing infertility due to absence of a (functional) uterus may now rightfully get hope by this fantastic news. Also the surgical community should be stunned by such technical accomplishment, though we invite you to look through the headlines, and see it as an exemplary demonstration how surgical innovation should be introduced.

The first report on the feasibility of uterine transplantation dates back to 2000 , though initially showing organ survival, unfortunately ending up with a hysterectomy for progressive necrosis after thrombosis of the uterine artery [2]. Later, the first first-trimester pregnancy was reported following transplantation from a brain-dead yet heart-beating donor [3]. From none of these two cases, there is prior track in the peerreviewed literature from preclinical experimentation or a comprehensive clinical research program. In contrast, the above achievement by Brännström et al. is the result of a strategy that meets the IDEAL-concept (innovation, development, exploration, assessment, long-term study) which should be used for the introduction of a novel treatment in a scientific and systematic way [4]. Gynecologic surgery wants to pay tribute to this program and summarizes below over 15 years of research on only few pages, found in the extensive literature generated by this group. Additionally, we want to congratulate the authors for reporting their success only after full achievement of what a fertility treatment is about: the taking home of a live baby.

\section{Definition of the target population and rationale}

Widely accepted and implemented transplantation procedures usually involve vital organs such as the heart, liver, or lung. Patients having undergone such transplantations have become pregnant, and while remaining safely under immunosuppressive 
medication, they had reasonably good outcomes. Transplantation of non-vital tissues or organs, such as the face, larynx, or limbs, is more recent and rather aims at improving the quality of life of the recipient. Uterine transplantation falls under this category, even if the World Health Organization considers infertility as a disease.

For these women with absolute uterine factor infertility, so far, the viable and recommended options for legal motherhood are at present either adoption or the even more complex bypass of gestational surrogacy with subsequent adoption to acquire legal motherhood [5]. Though viable, both solutions have major legal and psychological drawbacks, some of these would not apply if successful uterine transplantation would be possible and proof to be safe. Though the concept seems simple and logical, uterine transplantation is not a simple solution, as it exposes the recipient (future mother), if applicable also a living donor, and most importantly the (future) child to yet unknown risks.

Patients who may benefit from uterine transplantation either lack a uterus or have one that is functionally not compatible with pregnancy. Acquired conditions may compromise the uterine anatomy or function (e.g., synechia, fibroids) or even lead to hysterectomy (myomata, cancer, postpartum hemorrhage). Once there are no treatment options for those patients, and they meet the criteria for fertility treatment, uterine transplantation may be their only option apart from adoption or surrogacy. Some congenital anomalies are associated with the absence of an (adequate) uterus [6]. Of interest to reproduction in the latter patients, it is reassuring that genetic children born in a surrogacy program do not have the maternal condition their selves. If they were, this would be a serious limitation, if not an objection to uterine transplantation. When the transplantation is done for an acquired condition, there are usually less concerns about the individuals being good candidates for conception, unless perhaps in cancer patients with very poor prognosis, hence short life expectancy.

\section{Animal research steps and clinical program}

During the Brussels' conference, Mats Brännström took much of his speaking time to describe the experiments that made this surgical and medical challenge ready for clinical application [7]. A stepwise incremental approach was taken with experimental surgery in animals, going from lower animal species such as mice and rat to larger animals which size wise match more the clinical challenge, like sheep. Ultimately, the clinical scenario was successfully recapitulated in nonhuman primates, showing the relevance and need of primate research [8]. The group then launched a clinical trial (NCT01844362 www.clinicaltrial.gov) and recently reported on nine women with uteri being transplanted from live donors. Transplanted patients must attend frequent yet decreasingly clinical visits and laboratory testing. The clinical examination involves visual inspection of the transplanted uterine cervix, culture from the cervical canal, and occasional cervical biopsies to assess rejection. Criteria for rejection, the major drawback, were defined in the nonhuman primate experiments, once again underscoring the need for these in preclinical research [9]. Ultrasound scans with transvaginal and abdominal probes are done to assess uterine size, endometrial thickness and echogenicity, and uterine artery flow velocity waveforms.

Future gametes are obtained through in vitro fertilization (IVF). This is done before transplantation to ascertain that fertilization and initial embryo development is normal for the candidate couple. Also, IVF might be more difficult after transplantation because of the abnormal uterine location and nature of vascular pedicles and anastomoses. It also precludes post-pick up bleeding and ascending infection in an already immunosuppressed patient. Single embryo transfers are chosen to reduce the obstetrical risks.

The uterus transplantation is conceived as temporary. At the latest following completion of reproduction, it should be removed to avoid the side effects of persistent intake immunosuppressive agents. The current protocol is designed to allow maximum two pregnancies, or less if clinically indicated. Hysterectomy is foreseen sufficiently long after second delivery, at a moment the anatomy has returned to normal and the baby has reached a certain age.

Seven women had viable grafts with regular cycles, and a limited number of mild rejection episodes effectively reversed by short courses of increased immunosuppression. Success was, however, not universal. Two of the women underwent hysterectomy briefly after transplantation due to uterine artery thrombosis and intrauterine infection. The one patient now having delivered is a patient with Rokitansky syndrome. She has a single kidney and she previously obtained a functional vagina through self-dilatation. Through IVF, 11 embryos were obtained and cryopreserved. In 2013, she underwent uterine transplantation.

\section{Uterine transplantation}

The donor was a 61-year-old family friend, who herself had two children, confirming previous uterine "functionality." Persisting endometrial functionality was tested by administration of a contraceptive pill inducing regular bleeding patterns. In a 5-h operation, the uterus with large vascular pedicles, including the distal parts of the internal iliac veins and arteries, was removed. After extirpation, the uterus is flushed through the arterial ends and prepared for implantation.

One hour prior to the end of the above retrieval procedure, the operation in the future recipient started in an adjacent operating theater. Already, earlier induction immune 
suppressive agents were administered. Through a midline incision, the external iliac vessels were prepared for end-toside anastomosis. The blindly ending neovagina was separated from the bladder and rectum and opened. Sutures for later uterine fixation were placed bilaterally through the round ligaments, uterosacral ligaments, and the paravaginal connective tissues. Finally, the uterus was connected to the circulation, uterine veins first, followed by arteries where after blood flow to the uterus could be restored (Fig. 1). A Doppler probe was left for short-term surveillance of uterine artery flow; it was pulled out easily after a few uneventful days. The implantation lasted approx. $10 \mathrm{~h}$. The total ischemia time was around $140 \mathrm{~min}$.

Estimated blood loss for both patients was around 600$700 \mathrm{ml}$. Both recovered well; the recipient required a transfusion. Hospital stay was around 6 days. The recipient was given triple immunosuppression (tacrolimus, azathioprine, and corticosteroids). She developed two epochs of early rejection, reversed by steroids. She also required a conisation for a HPV infection picked up through cytologic changes, though donor and recipient were HPV-negative before.

One year later, she underwent a single embryo transfer, which resulted in a pregnancy. She kept working full time. During pregnancy, she was kept on low-dose prednisolone ( $5 \mathrm{mg}$ ), and followed up in the routine program for pregnant transplant patients, with visits every 2-3 weeks to specialists in high-risk obstetrics and transplantation. Ultrasound included follow up of the pulsatility index in the uterine and umbilical arter(y)(ies) and fetal growth surveillance, which remained within the (lower) normal limits, suggesting absence of increased resistance. The cervical length stayed also normal. She had another mild rejection during pregnancy reversed by additional steroids. Fetal growth was normal, but she developed suddenly severe preeclampsia in her $32 \mathrm{nd}$ week. She was admitted and given steroids for lung maturation. Because of fetal distress, an emergency cesarean section was done shortly after. Of note, her male baby was appropriately weighted for its age and did well without ventilatory assistance.

\section{Ethical aspects and implementation}

The ethical issues surrounding transplantation of non-vital organs are many. For uterus transplantation, they are even more complex and could so far only be theoretically approached. In contrast to most other transplantations, this strategy may require a live donor, which adds an additional risk to a non-beneficent of the procedure [10]. If outcomes would be as good with a deceased donor, then that could be preferred, because explantation can be done without risk and - this may be important to the outcome - with larger vascular pedicles.

Clinically, this and future pregnancies and their long-term outcome will be very useful for future risk assessment of the strategy. For instance, it is expected that preeclampsia will be more frequent because of the requirement of IVF and immunosuppression and the elder and allogenic uterus. The immunosuppressive drugs have their own potentially serious complications such as nephrotoxicity (tacrolimus), bone marrow toxicity (azathioprine), and diabetogenic effect (corticosteroids and
Fig. 1 Schematic drawing of the vascular anastomoses following uterine implantation. Reprinted with permission from the authors and the Lancet

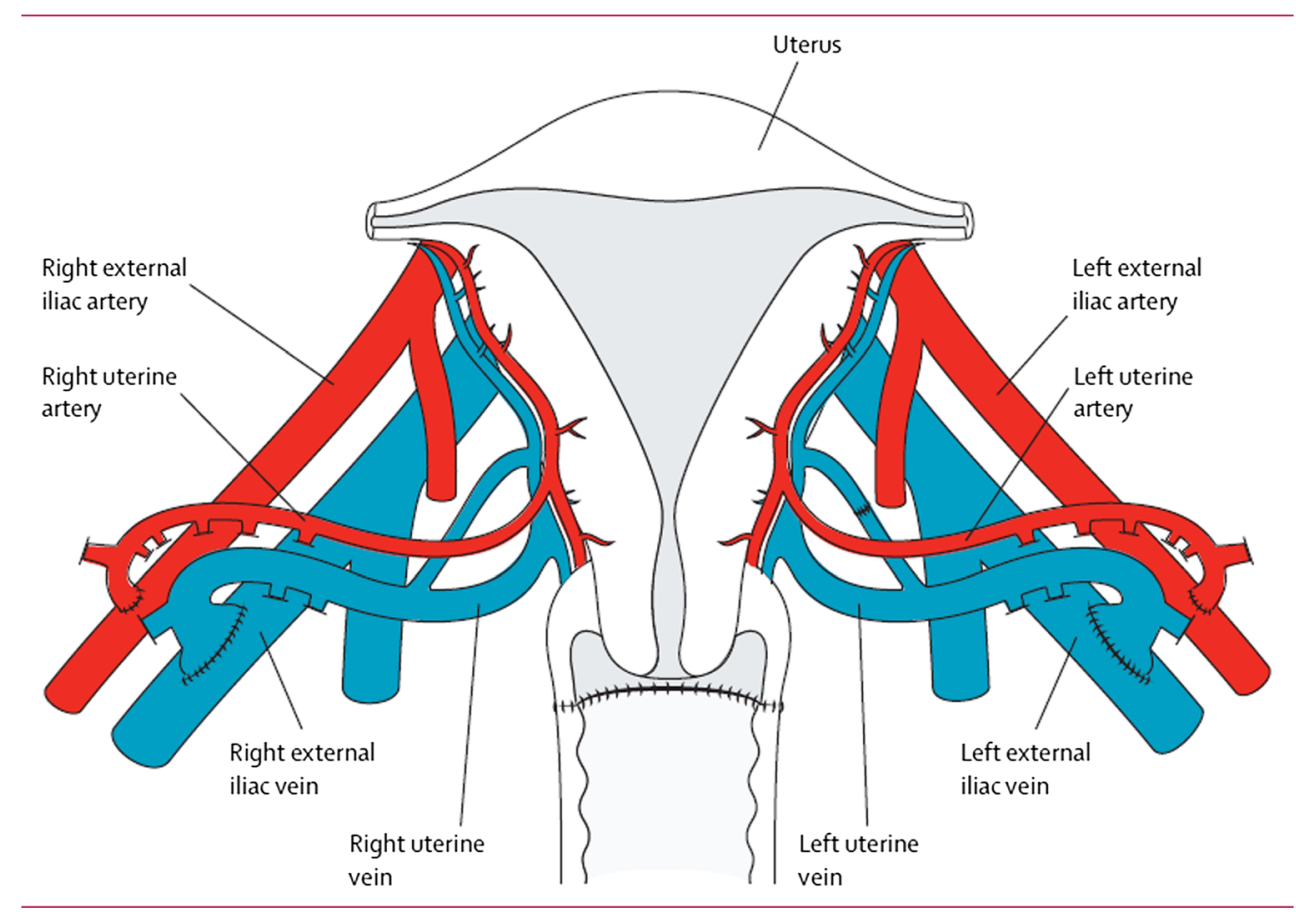


tacrolimus), as has been well documented in non-uterine transplantation patients. Another unique aspect of gestation in a transplanted uterus not directly related to the immunologic challenge is that it remains unknown how its vascular system remodels, what the eventual place of aspirin or likewise drugs would be, and what the consequences are of the lack of nerve regeneration (as far as we know).

This case will boost the further research, but the mediagenic character of that type of medical achievements may also have several side effects. This pregnancy, as well as the next ones to come, should for the time being only be conceived within a strict protocol and long-term follow-up program like the one currently in place at the Sahlgrenska Academy in Gothenburg. Potential beneficients may have to anxiously wait until a larger cohort of patients can be followed up by the Swedish team. In the early phase, it is also recommendable that patients are referred worldwide to the Gothenburg team, which is at present the only team with the required background, wider experience and skills, as well as a comprehensive program. Future surgical teams should explore the real need and potential volume they might achieve, then undergo extensive training in all aspects of uterine transplantation, yet not start clinical programs until the pioneers of the technique have reported safe reproducibility of this initial success in the peer-reviewed literature.

\section{References}

1. Bränström M et al (2014) Lancet. doi:10.1016/S0140-6736(14) 61728-1

2. Fageeh W, Raffa H, Jabbad H, Marzouki A (2002) Transplantation of the human uterus. Int J Gynaecol Obstet 76:245-251

3. Ozkan O, Akar ME, Ozkan O et al (2013) Preliminary results of the first human uterus transplantation from a multiorgan donor. Fertil Steril 99(2):470-476

4. McCulloch P, Altman DG, Campbell WB et al (2009) No surgical innovation without evaluation: the IDEAL recommendations. Lancet 374(9695):1105-1112

5. Del Priore G, Saso S, Meslin EM et al (2013) Uterine transplantation - a real possibility? The Indianapolis consensus. Hum Reprod 28(2):288-291

6. Grimbizis GF, Gordts S, Di Spiezio SA et al (2013) The ESHREESGE consensus on the classification of female genital tract congenital anomalies. Gynecol Surg 10(3):199-212

7. Brannstrom M, Diaz-Garcia C, Hanafy A, Olausson M, Tzakis A (2012) Uterus transplantation: animal research and human possibilities. Fertil Steril 97(6):1269-1276

8. Dancet EA, Spiessens C, Vangenechten R et al (2011) Acceptability of preclinical research on nonhuman primates in reproductive medicine: the patient perspective. Reprod Sci 18(1):70-78

9. Johannesson L, Enskog A, Molne J et al (2013) Preclinical report on allogeneic uterus transplantation in non-human primates. Hum Reprod 28(1):189-198

10. Olausson M, Johannesson L, Brattgard D et al (2014) Ethics of uterus transplantation with live donors. Fertil Steril 102(1):40-43 